\title{
Dementia Research Fit for the Planet: Reflections on Population Studies of Dementia for Researchers and Policy Makers Alike
}

\author{
Carol E. Brayne ${ }^{a}$ Linda E. Barnes ${ }^{a}$ Monique M.B. Breteler ${ }^{b, c}$ Rachael L. Brooks ${ }^{a}$ \\ Carole Dufouil $^{d}$ Chris Fox $^{\mathrm{e}}$ Laura Fratiglionif $^{f}$ M. Arfan Ikram ${ }^{g}$ Rose A. Kenny ${ }^{\text {h }}$ \\ Miia Kivipelto ${ }^{f}$ Antonio Lobo ${ }^{i}$ Massimo Musicco ${ }^{j}$ Chengxuan Qiu ${ }^{f}$ \\ Edo Richard $^{\text {k, I Steffi G. Riedel-Heller }}{ }^{m}$ Craig Ritchie ${ }^{n}$ Ingmar Skoog ${ }^{\circ}$ \\ Blossom C.M. Stephan ${ }^{p}$ Annalena Venneri ${ }^{q}$ Fiona E. Matthews ${ }^{r}$ \\ a Institute of Public Health, Department of Public Health and Primary Care, University of Cambridge, Cambridge, UK;

 \\ and Epidemiology (IMBIE), Faculty of Medicine, University of Bonn, Bonn, Germany; ${ }^{d}$ INSERM, Bordeaux, France; \\ e Faculty of Medicine and Health Sciences, University of East Anglia, Norwich, UK; ${ }^{f}$ Karolinska Institutet, Department of \\ Neurobiology, Care Sciences and Society, Stockholm, Sweden; ${ }^{9}$ Department of Epidemiology Erasmus Medical Centre, \\ Rotterdam, The Netherlands; ${ }^{\mathrm{h} C e n t r e ~ f o r ~ R e s e a r c h ~ i n ~ A g e i n g, ~ T r i n i t y ~ C o l l e g e ~ D u b l i n, ~ D u b l i n, ~ I r e l a n d ; ~ ' ~ U n i v e r s i t y ~ o f ~}$ \\ Zaragoza, Spain and Instituo Investigacion Sanitaria Aragon, Zaragoza, Spain; ${ }^{j}$ Institute of Biomedical Technologies, \\ National Research Council (ITB-CNR), Segrate, Milan, Italy; ${ }^{\text {} D e p a r t m e n t ~ o f ~ N e u r o l o g y, ~ D o n d e r s ~ I n s t i t u t e ~ f o r ~ B r a i n, ~}$ \\ Cognition and Behaviour, Radboud University Medical Centre, Nijmegen, The Netherlands; 'Department of Neurology \\ Amsterdam University Medical Centres, University of Amsterdam, Amsterdam, The Netherlands; ${ }^{\mathrm{m}}$ Institute of Social \\ Medicine, Occupational Health and Public Health, Medical Faculty, University of Leipzig, Leipzig, Germany; ${ }^{\mathrm{n} C e n t r e}$ \\ for Clinical Brain Sciences, University of Edinburgh, Edinburgh, UK; ${ }^{\circ}$ Department of Psychiatry and Neurochemistry, \\ Institute of Neuroscience and Physiology, University of Gothenburg, Gothenburg, Sweden; P Institute of Mental \\ Health, Nottingham University, Nottingham, UK; ${ }^{9}$ Department of Neuroscience, University of Sheffield, Sheffield, UK; \\ rPopulation Health Sciences Institute, Newcastle University, Newcastle, UK
}

\section{Keywords}

Guidelines · Population-based studies · Cohorts · Dementia group of European experts in population-based cohort research through a series of workshops, funded by the Joint Program for Neurodegenerative Disorders (JPND). The aims of the guidelines are to assist policy makers and researchers to understand (1) What population studies for ageing populations should encompass and (2) How to interpret the findings from population studies. Such studies are essential to provide evidence relevant to the understanding of healthy and frail brain ageing, including the dementia syndrome for contemporary and future societies by drawing on the past.

(c) 2020 The Author(s)

Published by S. Karger AG, Basel challenge. A set of guidelines have been developed by a

\begin{tabular}{ll}
\hline KARGER & (c) 2020 The Author(s) \\
Published by S. Karger AG, Basel & Targer \\
karger@karger.com & This article is licensed under the Creative Commons Attribution- \\
NonCommercial-NoDerivatives 4.0 International License (CC BY- \\
NC-ND) (http://www.karger.com/Services/OpenAccessLicense). \\
Usage and distribution for commercial purposes as well as any dis- \\
tribution of modified material requires written permission.
\end{tabular}




\section{Background}

\section{Why a Focus on Brain Ageing and Dementia?}

There has been a global increase in the age of our populations, resulting in a rise in the number of people affected directly and indirectly by dementia. The personal and socioeconomic burdens for dementia sufferers and their caregivers are significant as are the costs to society as a whole. Indeed, in 2008, the estimated cost of dementia in the EU was EUR 177.2 billion and by 2030 this figure is predicted to rise to over EUR 250 billion $[1,2]$. The global cost of dementia care in 2015 was estimated to be in the region of USD 818 billion, approximately 1\% of global gross domestic product with the largest proportion of this cost (70\%) being spent on informal, social, and direct medical care. It is for this reason dementia is a major public health challenge and has been recognized as a global public health priority by government and nongovernment (i.e., World Health Organisation and Alzheimer's Disease International) organizations [3].

\section{Context}

Why a Focus on Brain Ageing and Dementia?

In order to mitigate the forecasted dementia burden over the next 3 decades, from 50 million people in 2017 to 131.5 million by 2030 , there has been an increase in research into up stream prevention, early detection to delay or avert onset, and interventions to support those with dementia once the clinical syndrome is present. To achieve this requires a deep understanding of the nature of the dementia syndrome - in whom it occurs, what the risk profiles are, and what the evidence of impact is of any changes in natural history that might be effected through societal action or individualized interventions.

Substantial evidence on risk from population-based cohort studies has emerged suggesting that age-specific risk for dementia across generations has declined in high-income countries (including the USA, UK, and the Netherlands). There are also some promising findings from trials of interventions on risk profiles, although with limited impact. Through highly selected volunteer cohorts the ability to stage the neurobiological markers associated with clinicopathological Alzheimer's disease diagnosis is emerging with intensive phenotyping [4]. It is vital that policy makers, funders, and researchers understand the place of volunteer cohorts in the context of populationbased studies. Outputs from our Joint Program for Neurodegenerative Disorders (JPND) workshops set out the discussions and motivation for the creation of the guidelines for undertaking new cohort studies which form this paper.
Why Will the Guidelines Be Useful?

In the last decades, there has been an intense interest in the exact numbers of people in individual nations who "have dementia," with it becoming a mantra used by governments, charities, researchers, and businesses (e.g., "every $3 \mathrm{~s}$ globally a person develops dementia"). The question is, where do these data come from when they are not available from routine data in any country globally? Governments and various constituencies interested in, or with a need to, promote awareness of dementia rely on approximate or estimated numbers, guided by expert opinion, for prevalence (i.e., the number of cases of a disease or condition in a given population), incidence (the number of new cases of a disease or condition that occur over a defined period of time in a given population), natural history, and risk as almost always robust populationbased data are unavailable. Basing policy upon these estimates is likely, unless done with true knowledge of how the estimations are created, to result in a mismatch of resourcing with true need.

In the last few decades we have seen dramatic increases in life expectancy globally, with primary prevention of chronic conditions such as cardiovascular disease accounting for a significant portion of this increase. Despite this ageing of the population, recent population-based European studies, which have reported on dementia occurrence using stable diagnostic methods, suggest a significant age-specific reduction in dementia prevalence (although routine data-based studies reveal stability or an increase) [5-10]. This, along with rapidly changing diagnostic criteria, sociocultural environments, mass migration, and generational variation in life course experiences, means that governments can no longer assume that dementia itself and the needs of people living with dementia remain stable as new generations' age.

Global interest in dementia from research and biological angles over recent years has led to a rapidly increasing armamentarium of investigative methods and associated changes in diagnostic criteria, not just for manifest dementia but also for earlier phases of the underlying neurodegenerative diseases. Recent changes in these diagnostic criteria, in principle designed for research purposes, have incorporated biological measures into new diagnostic criteria which seek to define risk in people before the expression of a full dementia syndrome. The aim is better prediction of dementia and eventually early intervention strategies to decrease risk or prevent dementia onset. Approaches such as these are not without societal implications, for example, health, and life insurance. 
There is a need, indeed obligation, for societies and those who determine resource allocation, to invest in those policies on dementia care and prevention with the greatest likelihood of benefit for the population. Further, it is essential that such policies address social and gender inequality. This means that methods for risk stratification promoted for widespread use need to be fully tested in relevant populations, with some estimation of cost and benefit to truly understand risk prediction specific to the context it is to be applied. Development of such policies will require the integration of evidence from new approaches, testing against rigorous standards in relevant populations with detailed evaluation of emerging methods which currently include radioactive ligand imaging (potentially with multiple types of ligand and repeatedly across age).

These new approaches have largely been developed in highly selected volunteer and tertiary setting clinical populations. Accompanying these developments is a particular need to assess performance of any new emerging and sufficiently robust approach in population representative studies in order to assess their clinical utility, impact, harms, and costs.

\section{1st Century EURODEM Workshop Framing}

In 2015, a consortium of international dementia epidemiology experts was brought together under the JPND initiative "working groups to inform cohort studies". Prior to the initiation of the consortium, many of the members had actively contributed to the JPND remit and produced outputs to inform that call [11-14]. The first meeting of the consortium focused on cohorts and shared experience of investigators who have conducted population-based studies focused on cognition and dementia in Europe both historically (e.g., Cognitive Function and Ageing Study I [CFAS I], Cardiovascular Risk Factors Aging and Dementia and Gothenburg studies) and currently (e.g., Rhineland, LIFE, CFAS II). From these discussions, two themes emerged, Descriptive Epidemiology and Mechanistic Analysis.

Descriptive Epidemiology and its methods requires an assessment of definitions of disorders, purpose of diagnoses, the implications of a positive diagnosis, and the ability to synthesize findings across differing geographies, populations, and time. Epidemiology provides a context for health and social care provision at both national and local levels including resource allocation. It also supports, through the knowledge gained and numbers estimated, development of simple analytical models, which can be used to predict the future clinical, economic, and social

Dementia Research Fit for the Planet:

Reflections on Population Studies needs associated with neurodegenerative diseases and the possible impact of population change, whether through intervention or not. This is illustrated by CFAS in the UK, which has been used widely for estimating the future numbers of people with dementia as well as how these might be affected by changes in risk profiles at earlier ages.

Analysis to elucidate disease mechanisms, important for determining clinical outcomes, needs to take into consideration the methodological and analytical methods used to assess both risk and resilience factors. Attempts to stratify risk using new criteria for early disease stages with or without clinical symptoms have tended to assume that such factors are stable across time and populations an assumption that is not necessarily true, unless these have been tested in robust empirical analyses in a range of population-based samples. Risk stratification within clinical settings is seen as valuable; however, the evidence base to date is not yet sufficiently robust and has many shortcomings $[15,16]$.

In the population, cognition and cognitive change is a continuum. Over the last decades, attempts to pin down specific boundaries as to what constitutes dementia have created a moving field. The focus on dementia, without thinking about broader mechanistic analyses for brain failure, has created an emphasis which suggested that single proteins might be the answer to most dementia.

The recent strong move toward amalgamation of existing datasets, more or less harmonized, is conceptually attractive. Harmonization and combined analyses can however pose challenges, although traditional meta-analysis can be strengthened through consistent methodological approaches in the primary research cohorts themselves such as was the case with the earlier EURODEM linked incidence studies.

The creation of large samples for analyses may be possible through the pooling of datasets, however, ignoring their provenance is dangerous to later value. Once disconnected from knowledge of their generation, data can become misleading. The "Big Data" revolution, which includes vast amounts of data on individuals (-omics) as well as potential linkages to health and social care records and other internet data (purchases, movement, location), needs to be led by those with deep population engagement and understanding. This includes epidemiologists and experts within disciplines related to population health and well-being with support from bio-informaticians, machine learning/artificial intelligence experts, and the biomedical community. At present, efforts are not balanced in this way with the danger of heaping the 
rich rainbow of cohort resources into a single bucket of brown sludge from which little can be gleaned ("Rainbow in a Bucket").

Artificial intelligence is increasingly making its way into clinical practice (e.g., in diagnostics and imaging); however, its reliance on historical data, which are based on biased data generation or clinical practices, could create or perpetuate biases which may worsen patient outcomes. By strategically deploying AI and carefully selecting underlying data, algorithm developers can mitigate AI bias. Addressing bias could allow AI to reach its fullest potential by helping improvement of diagnosis and prediction while protecting patients [17].

Cohort studies can be a key contributor to the evaluation of novel diagnostic and prediction methods being developed in clinical settings. In order to understand the implications of rolling out such novel diagnostic methods, existing studies can provide a valuable base to test utility and pitfalls of implementation. Recent meta-analyses have highlighted inconsistent, and at times, poor methodological quality for validation of new diagnostics and prediction tools. In reaction to this, the Cochrane Collaboration developed the STARDdem criteria for diagnostic studies in dementia. A similar approach to quality assessment for prediction is also required for observational cohort studies specific to the array of potential risk and protective factors investigated as predictors from dementia cohorts [18].

In order to maximize investment in large studies without clinical diagnostic outcomes, but with well-measured cognition, particularly with regards to functional and social change, a good and integrated understanding of previously conducted research is required.

Historical investment in cohort studies of populations in Europe has undoubtedly strengthened the continent in the field of ageing (and dementia) research; however, it is apparent that there are still many unanswered and emerging questions. Prominent among these are whether dementia occurrence is changing as global population age is shifting; whether clinical profiles of dementia and their associated endobiological phenotypes are changing and will continue to change further; whether the relationship between diagnosis, disability, dependence, and survival as outcomes is changing; and what the interplay of relevant risk factors is at earlier life stages.

Uniquely placed to address these questions in a way that can be compared across time periods and geographies are population representative cohort studies. These are not simple to finance, design, run, and sustain. As such, there is a compelling need to both ensure maximi- zation of the value of existing data and argue for investment into specific new studies which can address questions relevant to contemporary and future populations at intermittent time periods, across geographies, and communities, most notably in areas where there is little or no contemporary or historical data.

These recommendations and guidelines aim to inform a wide range of audiences including researchers entering the area of dementia. They aim to assist dementia researchers understand what a population representative perspective is, where it fits, and critically, will provide the background framing for policy makers and funders who make decisions about the balance of research funding available to existing and new work, across biomedical and policy relevant domains. The guidelines cover all elements of the evolution of the dementia syndrome and cognitive impairment which does not meet criteria for dementia (whether mild cognitive impairment or the wider category of non-demented cognitively impaired).

\section{Basic Orientation}

What Are Population Representative Cohort Studies?

The definition of a population representative cohort study is not straightforward; here, the distinction between population derived and population representative can help (Table 1). While many cohort studies are population derived, relatively few are population representative.

Few volunteer cohorts across the life course have robust links to their original sampling frame; however, they have provided the bulk of our knowledge relating to risk factors for and the evolution of dementia within a given cohort. Without these links to the original sampling frame, such studies provide less evidence for the translation of findings back into the whole population of interest. Conversely, there are also limitations of population representative cohorts, which typically suffer from higher attrition rates than their volunteer counterparts and this, if not taken into account using appropriate biostatistical methods, can lead to biased findings on incidence rates and risk estimation.

What is meant by the word population and the relationship between personalized and collective approaches requires new scrutiny. This relationship is extremely important in the fields of dementia research and public health, as it is used as the basis for decision making regarding the balance of investment for current and future populations. There is a need in population descriptive 
Table 1. Population derived versus population representative

\begin{tabular}{lll}
\hline & Population derived & Population representative \\
\hline Sampling & $\begin{array}{c}\text { The original sample is drawn from a population, } \\
\text { no matter how unrepresentative the final sample is } \\
\text { after recruitment and subsequent follow-up waves }\end{array}$ & $\begin{array}{l}\text { - Recruitment is from a known population base. Can support purposeful } \\
\text { sampling from geographical areas or other defined clusters }\end{array}$ \\
\hline $\begin{array}{lll}\text { Key } \\
\text { features }\end{array}$ & - Volunteer studies examples include UK BioBank & $\begin{array}{l}\text { - Provenance of recruited sample is known and can be mapped back in a } \\
\text { robust statistical manner to the original population with appropriate } \\
\text { acknowledgement of potential bounds of uncertainty }\end{array}$ \\
& $\begin{array}{l}\text { Some information on the unseen population should ideally be available. } \\
\text { This can be in the form of aggregated contextual knowledge for the given } \\
\text { population as a whole }\end{array}$ \\
\hline
\end{tabular}

work to consider which groups are missed and underrepresented, even in studies that have been conducted with a view to be population representative.

Full exploration into research findings generalizability is rare, with most findings assumed to be generalizable from one setting to another. The dementia research field has an abundance of such examples; with many papers citing the need for their findings to be translated beyond the populations in which the evidence was generated. Sometimes these findings will be robust, often they will not. This lack of awareness of evidence provenance and generalizability is crucially important from a public health perspective and is likely to be a contributing factor in the repeated lack of replicability of findings in new samples. However, lack of replicability can also be attributable to genuine differences between populations across geographical areas and time periods; findings which could present new research questions [19].

Further critical areas of importance are the definitions of the original population sampling frame that is, who is, and who is not, included. This is particularly important for hard to reach or underserved, vulnerable populations and often includes those in care settings. Response rates and attrition are also critical in assessing the value of research in terms of generalizability and population meaning.

Since the development of genomic and biomarker measurement, the need for representative populations in studies has been neglected. Convenience samples are often studied without much attention being paid to selection bias. This does not mean population representation no longer matters. The poor performance of almost all studies of biomarkers when drawing on population approach is a major concern. The disconnection between tertiary clinic practice and performance in "usual" populations is not intuitive and poorly understood. It does not receive the attention it should, given the cost and poten-

Dementia Research Fit for the Planet:

Reflections on Population Studies tial harm to individual patients if tests developed on one population are applied to another in which the test has not been validated. A good example of this premature translation across settings from research to clinical might be amyloid imaging, which has not fared well in scrutiny of how robust it is through the Cochrane evidence review [20].

Current and future cohort studies, while highly valuable alone, can be further enhanced through the harmonization of data into a single structure for analyses. Harmonization is, however, extremely challenging and is more often an aspiration rather than achieved, other approaches are possible and can be more efficient [21]. It is important when designing new cohort studies to consider anchoring points to past studies and ensuring the use of consistent methodological approaches which will allow for harmonization and comparisons across time and space.

What is needed is strong support from policy makers and dementia researchers embarking on new work to integrate and interpret the gaps in current knowledge of the types and value of existing research, including epidemiological principles, approaches, and the role of harmonization and cross-study collaborations.

\section{What Are We Actually Studying and Why?}

In all research, including those making investments and those interested in current and future public health and population meaning we must always keep in mind the questions "what exactly is the thing we are studying?" and "how relevant is this to the societal concern of dementia and associated states in current and future populations?" In other words, if a new disease concept such as "pre-clinical Alzheimer's disease" leads to an epidemic of a diagnosis but one which has a benign prognosis, what does this mean for the societal "burden" of dementia and for the way in which an ageing society views itself? 
Dementia is a clinical syndrome, a valuable diagnostic label with a long history. Since diagnostic criteria were introduced these have moved from discursive texts to more specific lists of signs and symptoms. Early definitions that incorporated knowledge about vascular factors and ageing itself became replaced in the era of drug discovery by an emphasis on clinically diagnosed Alzheimer's disease, with its assumptions of pure underlying neuropathologies, and focus on particular proteins. More recently, this has been complemented by the rediscovery of vascular contributions to dementia, Lewy Bodies, and Parkinson's Disease, the multietiology of the relatively earlier onset frontal-temporal dementias and, most recently, the contribution of TDP-43 to not only frontal-temporal dementias but also hippocampal sclerosis. Knowing the extent to which diagnoses in cohort and specifically selected population studies differ from real life settings is crucial to determining the potential of any particular study to provide valuable insights into how risk reduction and prevention need to be tailored for populations, particularly those with the greatest occurrence of dementia.

Some have suggested that population studies of dementia are unnecessary, and that we can rely on clinical records or indeed forms of machine learning. If we had accurate, valid, reliable relationships between real time life and the data that are captured on each of us in many different places, including health and social care, this may be possible. However, this is not the case. Such data are biased or inaccurate, and it is essential that diagnostic ascertainment bias is considered if systems are to rely on routine data.

There are changes across time in the way in which diagnoses are made, and by whom. If diagnoses rely on clinical consensus and changing availability and interpretation of data, whether routine or from dedicated surveys, it is possible for diagnostic creep and boundary shifts to be introduced creating an illusion of change where there is none. In a study that is testing for change across time it is essential that the same symptoms give the same diagnoses for person number 1 and 1,000 measured at time 0 and 5 . The more invasive and less typical the diagnostic ascertainment the more likely that heterogeneity in diagnosis is removed, but at the cost of increased participant burden and attrition bias into diagnostic phases.

Different approaches to defining dementia adversely affect comparability of data across time and cross sectionally between, for example, geographies. In this regard, capturing social, functional, and cognitive outcomes and avoiding medical categorizations can overcome such inconsistencies although it introduces a welcome focus on what is meant by cognitive and functional decline, with the latter's relationship to context and comorbidity key too. Of greatest value are prospective cohort studies which apply consistent measurements of outcomes; however, cross-sectional studies are still of value if they accurately relate to a meaningful denominator [15].

Deep knowledge of population incidence and prevalence, including careful phenotyping across age, gender, education, culture and socioeconomic status, allows for better scrutiny of the research arising in more selective settings and the claims are made based on the results. Predictions can be tested with known outcome data and models improved. Biological metrics have been promoted to establish who in the population might be at risk of future dementia syndrome; however, these particular metrics cannot be assumed to either be sufficient to avert the dementia syndrome or indeed to replace knowledge of the lived life of individuals within their social and cultural contexts.

Taking a consistent approach to measurement allows trends in incidence and prevalence to be monitored. One way of doing this could be through the creation of a sentinel population in anchored clinical centers across Europe which collect a minimum core data set of longitudinal assessment from extant and newly created generational cohorts. In order to inform science and policy, as the cohorts represented at the workshop have already done, these would need to consider dementia development in the context of ageing, including noncognitive influences on morbidity in dementia, for example, social, physical, and economic factors as well as traditional cognitive approaches.

Cohorts with diverse populations allow for stratification and risk assessment that is robust. This in turn can be used either within cohort studies or in new population-based trials to test the potential for changes in trajectories. At present, the pressure to measure biological metrics in people with and without symptoms that might indicate early change has had a profound impact on some clinical services such that biomarker salience in diagnostic pathways is becoming the norm in settings, blurring the routine with the research agenda. This is clearly premature, with little evidence of real clinical value as yet. Demand has been driven by awareness, but without evidenced interventions or certainty in prognosis, undertaking such tests in large numbers of people is of poor value for health care investments where other evidenced opportunities exist to improve health and wellbeing.

As noted above new research criteria [22] have changed the boundaries used in a variety of diagnostic criteria re- 
lated to dementia (mild cognitive impairment with its changing definitions and Alzheimer's disease being key examples), and it is important to test systematically, and in relevant populations, the impact and meaning of such changes [23]. An example of the effect of new diagnostic criteria is that we do not know what the association and progression of novel biomarkers are in relation to disability and loss of independence. Population-based studies are essential to validate or refute their value and ensure that findings from the bench can be translated to the individual.

As well as the focus on the outcome of dementia, trends in the factors associated with risk for or protection from dementia are required from populations across the life course, for example, education and prevalence of smoking. Changes in these risk factors can be modeled for their impact on future dementia and the impact tested directly against the potential for therapeutics. Given the lack of effective treatments for any dementia, even those of early onset, such modeling can be done to test what would the impact of a medication have to be and at what age to reduce dementia risk by matching amounts to the life course risks already known $[24,25]$. New findings can then be scrutinized to see how they measure up to these known life course risks.

Although dementia as a syndrome can be analyzed as a single concept for certain purposes related to policy, health services, and public health, research does need to reflect its enormous heterogeneity. Researchers need to be aware of this heterogeneity, how the cohort studies represent dementia, and how their research fits into this. For cohort studies that aim to compare across geography and time, it is absolutely essential that there is consistency in approaches to study diagnoses, so that diagnostic drift and clinical shifts do not introduce systematic bias.

What Is the Role and Value to Society of Population

Representative Studies in Primary, Secondary, and

Tertiary Prevention? Setting the Agenda

The answer to this question hinges on the gaps in dementia research. While the research agenda is heavily influenced by those in clinical and biomedical fields, it is also determined by funders, politicians, and interested groups such as pharma, diagnostics, and technological innovation leaders, charities, patients, their families, carers, and the media. These constituents use epidemiological evidence of the size of the dementia "epidemic" or "tsunami" to justify particular activities. Rarely has there been evidence of reflection as to whether their promotional work actually maps onto the population evidence noted

Dementia Research Fit for the Planet:

Reflections on Population Studies above. An example is the fact that dementia is commonest in the oldest age groups, but that imaging studies are focused on the smaller populations at higher risk in younger age groups. Recommendations for clinical practice for all dementias are often based on knowledge from memory clinics and specialist centers ignoring the fact that most people with dementia have multiple comorbidities and would not pass through such clinical services.

One activity that attempted to counterbalance these perspectives is that of the James Lind Alliance [26]. This is a UK-based exercise exploring what the more general public consider as research priorities. It was developed in response to the fact that so little research actually addresses what people see as the need for evidence in many areas of challenges to health and wellbeing. The James Lind Alliance [27] is one in which the people, a much wider audience than general in terms of who drives the pattern of research funding and decision making, have a chance to pitch their perception on the questions unanswered by research. In the dementia-focused exercise, responses were distilled and synthesized into answerable questions, the literature searched on whether robust evidence exists and if not, these uncertainties were confirmed and a process of prioritization using established methodology followed. This process was conducted for primary, secondary, and tertiary prevention with the public and wider professional and institutional groupings. Over 4,000 individual questions were submitted, and all the questions were framed in terms of the public health prevention model [28]. Such exercises are being conducted in other countries too (e.g., Canada).

A public health research approach can meet these uncertainties as it is inherently multidisciplinary and relates to relevant populations and disorders in their societal context. Such work emphasizes the limited progress made with studies such as biomarkers in understanding their general limited specificity. Well-designed population representative cohort studies can address the urgent concern that, in pursuing the diagnostics currently being promoted in some clinical practices, we might be labeling a large group of people as suffering from a "disease" who would not develop symptoms in their lifetimes.

\section{Prevention}

\section{Primary Prevention}

The definition of primary prevention depends on perspective. If modifiable risk factors for the development of dementia are identified, and treatment of these will reduce 
risk of developing dementia, this would meet the definition of primary prevention. However, if this concerns biomarkers that are a marker of the earliest pathological manifestations in the development of a later clear-cut condition, such as cervical dysplasia, this is not primary but secondary prevention. We have compelling evidence of primary prevention at population level in many countries that has led to age-specific reduction of dementia risk [29], but without very clear knowledge of what life course factors contributed to/fostered this reduction.

Many countries now seek to include brain health in their health promotion messaging. This has encouraged an increase in individuals actively engaging in what they perceive to be risk-reducing activities such as physical, social, and cognitive activities. Balancing individualistic approaches with those for whole populations needs to be based on what benefit will be derived for whom. Individualistic approaches can further increase inequality, already apparent in dementia risk $[7,30]$. Such approaches are unlikely to be of value to less advantaged populations within and across countries. Trials and long-term natural history studies in diverse populations are important in this area to inform what can be done in whole populations, for particular types of societal and individual interventions [31]. Formal trials are often challenging in contexts in which the policy environment is changing, but it is important to strengthen methodologies that can evaluate natural experiments rigorously. Clinical conditions that constitute dementia risk are already being used in the UK health system ("health checks" now include dementia as an outcome for people with higher risk through conditions such as diabetes and stroke).

How do population cohort studies inform primary prevention research? Knowledge of whole populations informs trials, their designs, and their implementation. Population studies can also assist in the evaluation of primary prevention strategies through modeling and natural experiments (such as cohort changes). Estimation of treatment effects can be calculated using population data $[32,33]$, and population studies can inform primary prevention through adoption of interventions. Population studies also allow for quasiexperimental designs to be considered. For instance, the regression discontinuity design allows for estimation of treatment effects without the need for randomization [34].

Various risk factors (e.g., health and lifestyle risks) for dementia exert different effects throughout the life course, with potential cohort-effects depending on population characteristics, cultural context, and era in which data were collected. Research to support future prevention programs must cover the full spectrum of primary prevention, from targeting at risk groups to the whole population. Specific attention should be paid to cohort effects and geographical differences influencing the observational data on which future interventions will be based.

\section{Secondary Prevention}

Secondary prevention concerns any intervention intended to slow down neurobiological processes that are part of pathological pathways, and those interventions that reduce further cognitive decline in those with cognitive impairment not sufficient to merit dementia diagnosis. Biomarkers (CSF, imaging) and early clinical signs (such as neuropsychology or psychomotor disturbance) have the potential to be the measures that provide such detection (as in cancers). The marker needs to be extremely well understood and its relationship to the disorder in question must be very close indeed such that individuals (of particular age, sex, etc.) with particular metrics can be given their prognosis within reasonable bounds of uncertainty [35]. If biomarkers that can be obtained in a noninvasive, or minimally invasive way, have sufficiently good test characteristics (i.e., in terms of sensitivity, specificity, predictive accuracy, validity, and reliability, etc.) in the general population become available, and there are very effective treatments for that particular disease such that early detection changes natural history with much improved clinical outcomes it is conceivable that these could also be used for selecting the right target population for a secondary prevention program. This needs to draw on existing population cohort studies, to understand fully what the implications of the screening itself would be for the particular society.

Population data on natural history for biomarkers and clinical signs, their evolution, and relationship to outcomes of relevance to populations in which a disorder occurs are critical to progress the field of secondary prevention. The settings in which tests are used are extremely important, for example, prostate-specific antigen performs poorly in primary care but well in secondary and tertiary clinical settings. This requires knowledge of biomarkers in appropriate unselected populations to be studied using the proposed measures (usually "validated" in selective settings) on a repeated basis to see how well those who progress to develop a disorder within a population setting are identified by the proposed test or combinations of tests. Currently, even programs such as breast screening are being evaluated to attempt to make them less harmful to those who will not benefit. At present, a neglected consideration for secondary prevention 
Table 2. Prevention types

\begin{tabular}{|c|c|c|c|c|}
\hline Prevention type & Definition & Benefits & Considerations & Role of population studies \\
\hline Primary & $\begin{array}{l}\text { Upstream prevention } \\
\text { of a disorder or } \\
\text { disease } \\
\text { occurring }\end{array}$ & $\begin{array}{l}\text { Can be targeted toward whole } \\
\text { populations, communities, or at } \\
\text { individual levels } \\
\text { Can be targeted at high risk } \\
\text { populations based on clinical } \\
\text { characteristics }\end{array}$ & $\begin{array}{l}\text { Individualistic approach } \\
\text { to health that may lead } \\
\text { to a further increase in } \\
\text { health inequalities }\end{array}$ & $\begin{array}{l}\text { Data from population } \\
\text { studies contribute to } \\
\text { models about which risk } \\
\text { factors have the greatest } \\
\text { prevalence and potential } \\
\text { for societal and service } \\
\text { action } \\
\text { Without longitudinal data } \\
\text { on risk from population } \\
\text { representative studies for } \\
\text { given populations, the } \\
\text { true potential for primary } \\
\text { prevention cannot be } \\
\text { known }\end{array}$ \\
\hline Secondary & $\begin{array}{l}\text { The early detection of } \\
\text { a disease process } \\
\text { or the risk for a } \\
\text { disease progressing in } \\
\text { which the natural } \\
\text { history is understood } \\
\text { and well known }\end{array}$ & $\begin{array}{l}\text { Prevention/risk reduction } \\
\text { programs can be } \\
\text { opportunistically delivered or } \\
\text { delivered through screening } \\
\text { programs } \\
\text { With biological secondary } \\
\text { prevention the assumption } \\
\text { is that interruption of pathways } \\
\text { will be beneficial } \\
\text { at later life stages }\end{array}$ & $\begin{array}{l}\text { Consideration to } \\
\text { settings in which } \\
\text { tests are used are } \\
\text { extremely important } \\
\text { Justification must be } \\
\text { made regarding offering } \\
\text { biomarker screening to } \\
\text { an asymptomatic } \\
\text { population about the } \\
\text { harms and } \\
\text { benefits of any given } \\
\text { intervention }\end{array}$ & $\begin{array}{l}\text { Population-based studies } \\
\text { are essential for collecting } \\
\text { knowledge on how } \\
\text { secondary prevention } \\
\text { strategies should be } \\
\text { implemented }\end{array}$ \\
\hline Tertiary & $\begin{array}{l}\text { The mitigation of a } \\
\text { disorder once present }\end{array}$ & $\begin{array}{l}\text { Largely developed through trials } \\
\text { in clinical and other settings. } \\
\text { Also available on relatively small } \\
\text { numbers from within cohorts, } \\
\text { also includes end of life care }\end{array}$ & $\begin{array}{l}\text { Applying costings based } \\
\text { only on those seen in } \\
\text { services would not take } \\
\text { into account the full } \\
\text { range of need }\end{array}$ & $\begin{array}{l}\text { Population studies are } \\
\text { required to capture a } \\
\text { picture of the whole } \\
\text { population as it is in } \\
\text { society }\end{array}$ \\
\hline
\end{tabular}

is the nature of the evidence base required to justify offering biomarker screening to any asymptomatic populationsaboutharmsandbenefits ofanygiven intervention(s). In other words, what is the "offer" to society and an individual and what are the potential full costs (financial and other) of such an offer. All screening does harm, a fact that is relatively underrecognized [36].

Policy for secondary prevention programs must be based on robust evidence, and internationally accepted guidelines for such screening have been well described by the World Health Organisation, the US Preventative Task Force work, and the UK's National Screening Committee [37-39]. Up to date population-based studies, including cohort trials, are essential for knowledge about how secondary prevention should be implemented once preliminary trials have been conducted. Population evidence is crucial in the design of the research and policy development in this area.

Dementia Research Fit for the Planet:

Reflections on Population Studies

\section{Tertiary Prevention, Including End of Life}

Tertiary prevention means that for those people who have presented to health and social care, relevant and evidenced possibilities can be offered. The evidence base here is largely developed through trials in clinical and other settings, such as care homes and includes any intervention in those who have a diagnosis of dementia. It is a key that this also encompasses research about a dignified and compassionate approach to end of life care and decision making.

Population studies are again relevant as clinical settings do not capture the needs of the population as seen in the community. Individuals come and go from clinical services and are not then followed up to death. Indeed, primary care practitioners will not necessarily see those who are well supported within their communities. Research needs to describe the whole population, not just 
Table 3. Checklist for evaluation of population relevance

Original purpose

Details of the sampling frame, how it was generated, whether it is up to date, patterns of migration, and whether it includes care settings

Approach to participants, opt-in, opt-out, incentives, and exhortations

Details of the consent process

Response rates

Where people were interviewed, what they were offered. Uptake of different study offerings in terms of detailed phenotyping

What type of person did the interviews, how were they trained, evidence of quality control, and was clinical examination involved

Reasons for refusal within the study - gate keepers and proxies, individual refusal, specific refusal on questionnaire or investigation items, interviews abandoned

Ability to characterise the nonresponders at the population/geographical level

Attrition details (moving, death), over time

Further information on those who did not take part - complete or partial and if partial what are the characteristics of the people with missing data and the missing data themselves

Measures collected - origin of questions, validation if available (against what gold standard), whether coding and by design missingness exists

Do the measures include sufficient basic sociodemographic perspectives to provide contextual information for assessment of interpretation and generalisability?

Was the respondent interviewed alone? Were proxy informants involved?

Has there been stability of questions and measures across time? If not, how have questions changed and what is the potential implications of that change?

If biological samples were collected, are they appropriate to the questions being addressed? This includes the way they were taken, who by and how were they handled (e.g., immediately and shipped to storage, stored, extracted, and analysed)

How have problematic areas such as different IADL and ADL for men and women through cultural disability been explored

If routine records were used in follow-up what was the health system at the time and what biases/influences could have been introduced for example, health service and social policy change?

Have any or all of these factors been examined in any outputs to date? If yes, is it only to say these might introduce bias or limitations or is there further exploration to test which way the bias might go and how large it might be?

Have the findings been contextualized using all this information to assess what the true value of the outputs are? This means for that population, in relation to other known, nonindependent risk factors and the potential for population impact is (i.e., meaningful effect sizes rather than $p$ values)? This is in contrast to limited publication of narrow associations that do not provide context and meaning

ADL, activities of daily living; IADL, instrumental ADL. those seen in particular settings in order to understand the reality of dementia in populations. Applying costings based only on those seen in services to all people with dementia does not take into account the full range of need and may well be severely impacted by the unique characteristics of the individuals in such studies. The complete picture of the natural history and cost of dementia may therefore be inaccurately estimated. Such research informs policy development in an important and unique way, relevant to work such as the UKs NICE assessments (and similar ones elsewhere in Europe and beyond). Population-based studies provide a background and a potential resource in which research aimed at tertiary prevention can be developed. Trials that work with this kind of approach are more likely to be relevant to the ultimate aim of all health research to improve lived lives for the population (Table 2 ).

\section{Guidance on Understanding Existing Data}

In order to conduct truly informative integrated analyses with cohort data for the purposes of addressing the areas noted above, the following areas as outlined in the checklist in Table 3 must be well understood. Quality of reporting in these areas is highly variable, but it is increasingly recommended by journals that this is improved.

\section{Commentary}

Most studies will not be able to meet the highest standards in all of the areas outlines above. However, some attempt is warranted, and any findings presented without sensitivity analyses to explore the impact of inevitable imperfection of all studies should be treated with caution. This is because the study will not be able to contribute fully to synthesized approaches about whole populations.

There will be internally valid questions that can be answered without going through this challenging list, but these will be divorced from true populations and thus their meaning for policy and future populations will be unclear.

\section{Guidance on Repurposing and Establishing New Cohorts to Address the Areas Above}

If you are planning on establishing a new cohort, first of all, assure yourself that the planned new cohort will truly have added value to answer specific research ques- 
tions. Second, consider if you will have sufficient power to answer your specific research questions. Existing cohorts across generations still have great value to generate and test hypotheses about causation, natural history, and the relationship between ageing and dementia. However, this must be done with nuanced understanding of cohorts.

In addition to maximizing the policy and scientific value of existing cohorts, as implicit from the discussion above, new cohorts are urgently needed for those geographical areas and social groups where data are decades out of date or no data exist. This is of particular concern to Eastern Europe and low- and middle-income countries, which have substantial dementia burdens, but very limited research and health care capacity to deal with their ageing populations. Some cohorts will have the potential to incorporate experimental and interventional approaches or be able to be used to evaluate geographical changes. This has a range of implications and considerations for which new guidelines need developing.

All elements in the above checklist (Table 3 ) should be integrated into the design and collection of data at each study wave. Additionally, and depending on context, there should be detailed consideration of how inequalities and hard to reach communities are being included with particular design features appropriate to culture and setting (this will include gender and location of study being conducted for some communities). Our core recommended template for undertaking new cohort studies is shown in Table 4.

\section{Guidelines on Research Expertise Requirements}

\section{Skill Requirements for Cohorts}

Undertaking a new cohort study requires a multidisciplinary team committed to the long-term gathering of data and attainment of the highest possible response rate based on the decisions taken, whatever the chosen sample size. The more a study's inclusion criteria are based on onerous and detailed outcome measures, the lower the response rate for a complete population is likely to be. Studies require expertise in public health, statistics, general practice, specialist medical areas, schools of behavioral science, study coordinators and managers, interview trainers (if appropriate), as well as professionals skilled in communication, IT (if applicable), ethics, and data handling. Many skills associated with the set up or following of a cohort study are not discipline specific and
Table 4. Guidelines for undertaking new cohort studies

Aim: Could the question be addressed through existing cohorts or other approaches?

Design of study

Knowledge of the population denominator, in as much detail as allowed prior to sampling (and usually consent)

Ability to understand reasons for refusal from all participants (as much detail as can be allowed within ethical considerations)

Options for levels of participation (clinic visit, home visit, internet response, informants, medical/social care record access) to increase participation rates

Considerations of enticements or remuneration for participants

Covering sociodemographic features and migration within and across regions and countries

Special undertakings for underrepresented populations

Capacity and consent and how to maximize response

Interval of change, how much and how often?

Development of strategies to minimize loss to follow-up

Measurements: Will there be sufficient power to address key questions?

Core elements that can be compared across all new studies are the key here, such as gender, age, social-economic circumstances, chronic diseases, functional impairments, interactions with care givers (both formal and informal), and cognitive testing (at least broad). Main aspects must have been measured (and reported on) in exactly the same way from at least one other study (in the geographical region) to provide anchoring of measurements over time. This anchoring can be different studies (e.g., measurement of education is the same as UK Census, whereas functional impairment is the same as another study) but exact replication is the key

Measuring risk and compensatory factors across the life course, including vascular history and status along with other relevant medical conditions

Measuring regular medications, interactions with health services and care

Potential to measure easily accessible biological material, for example, hair or nails collected appropriately

Questionnaires online or in person, in clinics, or combinations

Potential for computerized testing in addition to existing and well-understood testing procedures (not instead of an anchoring measure)

Subsets with deeper phenotyping, deeper risk/protection data collection. Random subsets with known linkage. Not opportunistic, but designed to be maximally informative 
therefore not taught through usual channels. Career development can be seriously inhibited for those involved in the set up and follow-up of cohorts unless teams are well resourced.

\section{Capacity Building for the Future}

As stated above, the core skills needed for undertaking cohort studies are rarely taught in any formal arena. Years of expertise and knowledge have been captured in this contribution by the leading researchers around Europe (and internationally) who are at the forefront of this type of research. Capacity building presently relies on these research groups to fund, and train, new individuals to be the next generation of population-based researchers. Yet funding does not always allow for such a transition. It takes years for a study to reach maturity from initiation, mostly without core capacity involvement, reliant on single leaders bringing together committed teams over prolonged periods. New areas need developing to meet contemporary challenges, as well as to enhance the value of this type of study, including integration of routine data, biomedical methodology for big data and incorporation of new types of such data in an informative manner (e.g., geospatial, atmospheric, economic, and commercial). Few outside these communities appreciate the detailed issues outlined here. Sadly, on the basis of contemporary investment into dementia research $[33,35]$, this is likely to lead to considerable future wasted investment in research avenues which have not been scrutinized fully nor evaluated for population relevance and potential for benefit. Training basic scientists and policy makers to be able to appraise critically (but fairly) evidence from all sources gives additional capacity to the community in terms of understanding population health.

\section{How to Use These Guidelines}

\section{For Funders}

Read and think about what the balance of your funding is and training for such skills. Long-term vision is required not only for new cohorts, but in particular for future prevention trials.

\section{Policy Makers}

Read and apply the evidence provided to you which appears to be compelling, and question who and where did it come from? What constituencies stand to gain and over what time scale?

\section{Journal Editors and Reviewers}

Read and think about what you publish, much research is not contextualized and its value is limited because such careful considerations are not made. Further, reviewers are not trained in this and should also take note. This will lead to higher quality publications and greater use of the excellent guidelines on publication standards $[16,39]$.

\section{Steward of a Cohort which Might Be Population \\ Representative}

Think about whether you could address some of the areas where your study is weaker as there are often other sources of information that can help with contextual value. Think before you overplay your results without consideration of impact.

\section{Cohort Investigator Hopefuls, Applying for Funds}

Advise against new cohorts unless you are very clear what this new cohort adds to existing cohorts, that is, a new era is perhaps not necessarily a strong enough argument to start a new cohort. Very clear research questions are required. Study designs should be determined by a statistical power calculation for the main research questions that the cohort would seek to address (as opposed to just collecting as much data as possible to fill a biobank).

Aim for (inter)national collaboration to start few major cohorts, rather than multiple smaller cohorts. Focus on research questions. Measure parameters for these specific research questions in depth, rather than measuring a very broad range of parameters.

Other Dementia Researchers of a Non-Population

Representative Cohort or If You Work in Primary,

Secondary, and Tertiary Care

Do not translate data of a nonrepresentative sample to a wider population without very careful consideration of the pitfalls. Aim for proof of concept analyses in highly selected populations. Never lose the scope of your cohort: highly selected, limited external validity. Look at your study through the lens of this guidance and see whether you can meet any of the elements.

\section{Members of the Public or a Charity}

Question received statements about the evidence, ask where it came from, and how appropriate it is for the challenges that society grapples with. 


\section{Discussion}

This paper synthesizes a unique expert group's knowledge about a key area for society based on centuries of collective experience of ageing populations and dementia. The key messages for researchers and policy makers alike working in the area of brain ageing and dementia research are that there is no substitute for research, including those studies that are conducted across many decades in contrast to other designs that are necessarily short term (e.g., RCTs), which has a population grounding if research is to fully benefit further generations.

We envisage that these guidelines will be of use to researchers and policy makers to assess the value of existing and new cohort studies and determine what is required to carry out and interpret findings relevant to healthy and frail brain ageing, including the dementia syndrome in populations. The guidelines provide a framework to ensure that the highest standard of research can be attained.

There is a need to utilize and maximize current and future investments into cohort studies in order to generate greatest benefit to the public and scientific community alike. It is hoped that these guidelines will maximize these investments through the considered understanding of existing data, repurposing of cohorts, and establishment and efficient design of future cohorts that include anchoring data points to past cohorts and enabling harmonization of data across studies, geographies, and time points which will translate into a robust data source for future research.

These guidelines were developed by a consensus group of experts from across Europe and represent the opinions of the participants. The consensus group was funded by the JPND and was developed through a series of workshops.

\section{Acknowledgments}

We would like to thank the Karolinska institute for hosting the grant (EU-FORTE project dnr. 2014-5166).

\section{Author Contributions}

All authors attended the discussion workshops and contributed fully to the generation of the guidelines proposed. C.E.B. and R.L.B. wrote the manuscript, while all authors provided comments and edits to the text before approving the final manuscript.

\section{References}

1 Wimo A, Jönnson LG. Cost of illness and burden of dementia - The base option. [Accessed 14 June 2017]. Available from: http://www. alzheimer-europe.org/Research/EuropeanCollaboration-on-Dementia/Cost-of-dementia/Cost-of-illness-and-burden-of-dementia.

2 Wimo A, Jönnson L. G. A. Prognosis to 2030 - Cost of dementia - European Collaboration on Dementia - Research - Alzheimer Europe. [Accessed 14 June 2017]. Available from: http://www.alzheimer-europe.org/Research/ European-Collaboration-on-Dementia/ Cost-of-dementia/Prognosis-to-2030.

3 WHO. WHO|Dementia: a public health priority. WHO (2016). [Accessed 14 June 2017]. Available from: http://www.who.int/mental_ health/publications/dementia_report_2012/ en/.

4 Hanseeuw BJ, Betensky RA, Jacobs HL, et al. Association of Amyloid and Tau with Cognition in Preclinical Alzheimer Disease: A longitudinal Study. JAMA Neurol. 2019, Epub ahead of print.

5 Skoog I, Börjesson-Hanson A, Kern S, Johansson L, Falk H, Sigström R, et al. Decreasing prevalence of dementia in 85-year olds examined 22 years apart: the influence of education and stroke. Sci Rep. 2017 Jul;7(1):6136.

6 Lobo A, Saz P, Marcos G, Día JL, De-la-Cámara C, Ventura T, et al. Prevalence of "organic brain syndrome" in a Southern European population in two different time periods. The ZARADEMP Project. Eur J Psychiatry. 2005; 19(2):112-9.

7 Matthews FE, Arthur A, Barnes LE, Bond J, Jagger C, Robinson L, et al.; Medical Research Council Cognitive Function and Ageing Collaboration. A two-decade comparison of prevalence of dementia in individuals aged 65 years and older from three geographical areas of England: results of the Cognitive Function and Ageing Study I and II. Lancet. 2013 Oct; 382(9902):1405-12.

8 Qiu C, von Strauss E, Bäckman L, Winblad B, Fratiglioni L. Twenty-year changes in dementia occurrence suggest decreasing incidence in central Stockholm, Sweden. Neurology. 2013 May;80(20):1888-94.

9 Schrijvers EM, Verhaaren BF, Koudstaal PJ, Hofman A, Ikram MA, Breteler MM. Is dementia incidence declining?: trends in dementia incidence since 1990 in the Rotterdam Study. Neurology. 2012 May;78(19):1456-63.

10 van Bussel EF, Richard E, Arts DL, Nooyens AC, Coloma PM, de Waal MW, et al. Dementia incidence trend over 1992-2014 in the Netherlands: Analysis of primary care data. PLoS Med. 2017 Mar;14(3):e1002235.

11 DSM-IV and DSM-5 criteria for dementia. [Accessed 14 June 2017]. Available from: https://www.uptodate.com/contents/ image?imageKey=NEURO/91276.

12 Breeze E, Hart NJ, Aarsland D, Moody C, Brayne C. Harnessing the power of cohort studies for dementia research. J Public Ment Health. 2015;14(1):8-17.

$13 \mathrm{Wu}$ YT, Fratiglioni L, Matthews FE, Lobo A, Breteler MM, Skoog I, et al. Dementia in western Europe: epidemiological evidence and implications for policy making. Lancet Neurol. 2016 Jan;15(1):116-24.

14 JPND. Longitudinal cohort studies in neurodegeneration research. (2013). [Accessed 14 June 2017]. Available from: http://www. neurodegenerationresearch.eu/initiatives/ jpnd-alignment-actions/longitudinal-cohorts/.

15 Bennett DA, Brayne C, Feigin VL, BarkerCollo S, Brainin M, Davis D, et al. Development of the standards of reporting of neurological disorders (STROND) checklist: a guideline for the reporting of incidence and prevalence studies in neuroepidemiology. Eur J Epidemiol. 2015 Jul;30(7):569-76.

16 Weuve J, Proust-Lima C, Power MC, Gross AL, Hofer SM, Thiébaut R, et al.; MELODEM Initiative. Guidelines for reporting methodological challenges and evaluating potential bias in dementia research. Alzheimers Dement. 2015 Sep;11(9):1098-109. 
17 Parikh RB, Teeple S, Navathe AS. Addressing Bias in Artificial Intelligence in Health Care. JAMA. 2019 Nov;9(24):2377.

18 Noel-Storr AH, McCleery JM, Richard E, Ritchie CW, Flicker L, Cullum SJ, et al. Reporting standards for studies of diagnostic test accuracy in dementia: The STARDdem Initiative. Neurology. 2014 Jul;83(4):364-73.

19 Bak TH. Cooking pasta in La Paz. Linguist Approaches Biling. 2016;6(5):699-717.

20 McShane R, Malouf R, Collins H, Ritchie CW. Diagnostic test accuracy in dementia: A programme of systematic cochrane reviews. Alzheimers Dement. 2009;5(4):P2778.

21 Griffith L, van den Heuvel E, Fortier I. Agency for Healthcare Research and Quality (US); 2013 Mar. [Accessed 4 December 2019].

22 Dubois B, Hampel H, Feldman HH, Scheltens P, Aisen P, Andrieu S, et al.; Proceedings of the Meeting of the International Working Group (IWG) and the American Alzheimer's Association on "The Preclinical State of AD"; July 23, 2015; Washington DC, USA. Preclinical Alzheimer's disease: Definition, natural history, and diagnostic criteria. Alzheimers Dement. 2016 Mar;12(3): 292-323.

23 McCleery J, Flicker L, Richard E, Quinn TJ. The National Institute on Aging and $\mathrm{Al}$ zheimer's Association research framework: A commentary from the Cochrane Dementia and Cognitive Improvement Group. Alzheimers Dement. 2019 Jan;15(1):179-81.

24 Norton S, Matthews FE, Barnes DE, Yaffe K, Brayne CE. Potential for primary prevention of Alzheimers' disease: an analysis of population-based data. Lancet Neurol. 2014 Aug; 13(8):788-94
25 Livingston G, Sommerlad A, Orgeta V, Costafreda SG, Huntley J, Ames D, et al. Dementia prevention, intervention, and care. Lancet. 2017 Dec;390(10113):2673-734

26 Kelly S, Lafortune L, Hart N, Cowan K, Fenton M, Brayne C; Dementia Priority Setting Partnership. Dementia priority setting partnership with the James Lind Alliance: using patient and public involvement and the evidence base to inform the research agenda. Age Ageing. 2015 Nov;44(6):985-93.

27 The James Lind Alliance. [Accessed 3 December 2019]. Available from: http://www.jla. nihr.ac.uk/.

28 Society A. Outcomes from the James Lind Alliance priority setting partnership - Alzheimer's Society. (2015). [Accessed 14 June 2017]. Available from: https://www.alzheimers.org. uk/site/scripts/download_info.php?fileID= 2226.

29 Richard E, Moll van Charante EP, Hoevenaar-Blom MP, Coley N, Barbera M, van der Groep A, et al. Healthy ageing through internet counselling in the elderly (HATICE): a multinational, randomised controlled trial. Lancet Digital Health. 2019;1(8):E42434.

30 Matthews FE, Stephan BC, Robinson L, Jagger C, Barnes LE, Arthur A, et al.; Cognitive Function and Ageing Studies (CFAS) Collaboration. A two decade dementia incidence comparison from the Cognitive Function and Ageing Studies I and II. Nat Commun. 2016 Apr;7(1):11398.

31 Stephan BCM, Birdi R, Tang EYH, Cosco TD, Donini LM, Licher S, et al. Secular Trends in Dementia Prevalence and Incidence Worldwide: A systematic review. J Alzheimers Dis. 2018;66(2):653-80.
32 Matthews FE, McKeith I, Bond J, Brayne C; MRC CFAS. Reaching the population with dementia drugs: what are the challenges? Int J Geriatr Psychiatry. 2007 Jul;22(7):62731 .

33 Matthews FE, Muniz-Terrera G, McKeith I, Brayne C; MRC CFAS. Who will be eligible? An investigation of the dementia population eligible for cholinesterase treatment following the change in NICE guidance. Int J Geriatr Psychiatry. 2010 Jul;25(7):719-24.

34 van Leeuwen N, Lingsma HF, de Craen AJ, Nieboer D, Mooijaart SP, Richard E, et al. Regression Discontinuity Design: Simulation and Application in Two Cardiovascular Trials with Continuous Outcomes. Epidemiology. 2016 Jul;27(4):503-11.

35 Wald NJ, Hackshaw AK, Frost CD. When can a risk factor be used as a worthwhile screening test? BMJ. 1999 Dec;319(7224):1562-5.

36 Gray JAM, Patnick J, Blanks RG. Maximising benefit and minimising harm of screening. BMJ. 2008 Mar;336(7642):480-3.

37 Alzheimers Society. Dementia research roadmap for prevention, diagnosis, intervention and care by 2025. [Accessed 3 December 2019]. Available from: https://www.alzheimers.org.uk/research/for-researchers/researchroadmap.

38 Pickett J, Brayne C. The scale and profile of global dementia research funding. Lancet. 2019 Nov;394(10212):1888-9.

39 von Elm E, Altman DG, Egger M, Pocock SJ, Gøtzsche PC, Vandenbroucke JP; STROBEInitiative. [The Strengthening the Reporting of Observational Studies in Epidemiology (STROBE) statement: guidelines for reporting of observational studies]. Internist (Berl). 2008 Jun;49(6):688-93. 